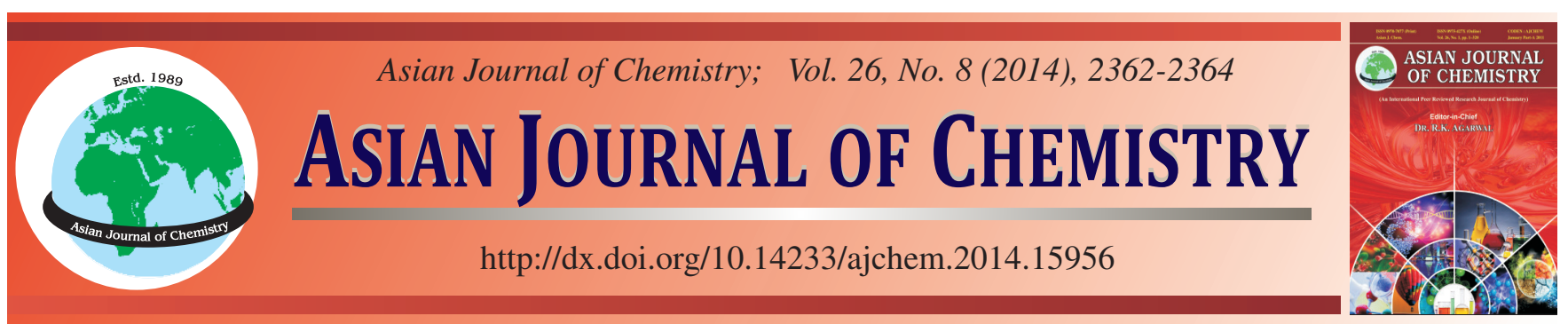

\title{
Synthesis and Evaluation of Some Substituted Heterocyclic Fluconazole Analogues as Antifungal Agents
}

Shudong Wang ${ }^{1, \dagger}$, Lei Zhang ${ }^{2,3, \dagger}$, Yongsheng Jin ${ }^{2}$, Jin Hao TAng ${ }^{1}$, Hua Su ${ }^{1}$, Shichong Yu ${ }^{2, *}$ and Haixiang Ren ${ }^{1, *}$

${ }^{1}$ Department of Pharmacy, General Hospital of Nanjing Military Region, Chinese People's Liberation Army, Zhongshan East Road 305, Nanjing 210002, P.R. China

${ }^{2}$ College of Pharmacy, Second Military Medical University, Guohe Road 325, Shanghai 200433, P.R. China

${ }^{3}$ College of Pharmacy, Yantai University, Qingquan Road 30, Yantai 264005, P.R. China

*Corresponding authors: E-mail: yuscc1008@163.com; haixiangreng@163.com

$\uparrow$ Shudong Wang and Lei Zhang contributed equally to this work

A new series of fluconazole analogues of 1-(1H-1,2,4-triazol-1-yl)-2-(2,4-difluoro-phenyl)-3-4-(substituted-heterocyclic ring-1H-1,2,3triazol-1-yl)-2-propanols (1-10) were designed, synthesized and evaluated as antifungal agents. Preliminary antifungal tests showed that most of the title compounds exhibited moderate activity with broad spectrum against eight human pathogenic fungi in vitro, compounds $\mathbf{1}$ and $\mathbf{6}$ had the best antifungal activity against Candida albicans with the value of $\mathrm{MIC}_{80}=0.5 \mu \mathrm{g} / \mathrm{mL}$ respectively.

Keywords: Triazole, Synthesis, Antifungal activity, 1,3,4-Oxadiazol.

\section{INTRODUCTION}

During the past few decades, fungal infections are the commonly prevalent diseases from which a large proportion of the human population suffers ${ }^{1-5}$. The increased emergence of both the superficial and systemic fungal infections has led to the massive increase in the rate of mortality, especially in the immuno-compromised individuals, those suffering from tuberculosis, cancer or AIDS ${ }^{6,7}$. In clinic, azoles, especially triazole antifungal agents, were used most widely and efficiently. For example, fluconazole, voriconazole and itraconazole, presently play an important role in the treatment of fungal infections. However, their clinical use has been limited by the emergence of drug resistance, high risk of toxicity, insufficiencies in their antifungal activity and undesirable side effects ${ }^{8-10}$. Hence, there is still a need to develop the safe and efficient chemotherapeutic agents with potent broad spectrum antifungal activities ${ }^{10}$.

\section{EXPERIMENTAL}

Analytical TLC was carried out on Silica Gel 60 F254 plates (E. Merck) with detection by fluorescence and/or by charring with $10 \%(\mathrm{v} / \mathrm{v}) \mathrm{H}_{2} \mathrm{SO}_{4}$ in EtOH. Column chromatograph was performed on silica gel (200-300 mesh, Qindao Ocean Chemical Co., China). All chemicals and solvents were purchased from commercial sources unless specified otherwise. ${ }^{1} \mathrm{H}$ and ${ }^{13} \mathrm{C}$ NMR spectra were recorded in $\mathrm{CDCl}_{3}$, using
TMS as internal standard NMR solvent. Mass spectra were performed on an API-3000 LC-MS and Agilent Q-TOF-MS spectrometer.

Synthesis of target compounds: The general synthetic methodology for the preparation of title compounds is outlined in Scheme-I. The key intermediate $\mathbf{1}$ was synthesized by the reported procedure ${ }^{11,12}$, the triazolone-containing side chains were prepared via four steps. Various substituted benzoic acid 1-10a were converted to methyl benzoate $\mathbf{1 - 1 0 b}$ by reacting with methanol, followed by treating with hydrazine hydrate to give benzohydrazide 1-10c. 1-10d were synthesized by reacting the 1-10c with carbon disulphide in presence of $\mathrm{KOH}$ and conc sulfuric acid and then reacted with 3-bromo-1propyne to give the side chains 1-10e. The compounds 1-10 were carried out under the click-reaction ${ }^{13,14}$ condition in the presence of $\mathrm{CuSO}_{4}$ and sodium ascorbate. All the title compounds (1-10) described above were characterized by ESI and NMR spectroscopic analysis.

Compound 1: Yellow oil, yield: $81 \%$; ${ }^{1} \mathrm{H}$ NMR (300 $\left.\mathrm{MHz}, \mathrm{CDCl}_{3}\right) \delta 8.02(\mathrm{~s}, 1 \mathrm{H}), 7.94-7.73(\mathrm{~m}, 3 \mathrm{H}), 7.50-7.26$ (m, 4H), 6.78-6.70 (dd, $2 \mathrm{H}, J=16.8,7.3 \mathrm{~Hz}), 5.35(\mathrm{~s}, 1 \mathrm{H})$, 4.89-4.84 (d, $1 \mathrm{H}, J=14.2 \mathrm{~Hz}), 4.75-4.72(\mathrm{~m}, 2 \mathrm{H}), 4.63-4.46$ (m, 2H), 4.33-4.29 (d, 1H, J = 14.3 Hz), 2.69 (s, 3H); [M $+\mathrm{H}]^{+}$511.14, found 511.57.

Compound 2: Yellow oil, yield: $83 \%$; ${ }^{1} \mathrm{H}$ NMR (300 $\left.\mathrm{MHz}, \mathrm{CDCl}_{3}\right) \delta$ 8.16-7.67 (m, 5H), 7.41-7.39 (m, 3H), 6.72- 
<smiles>NCC(O)(CN)c1ccc(F)cc1F</smiles>

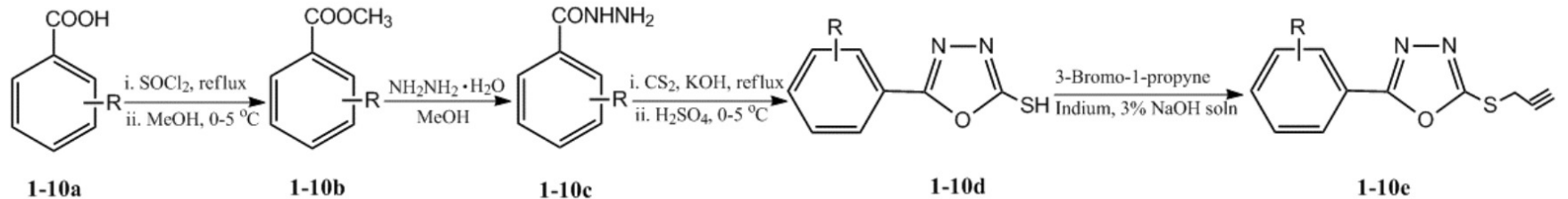

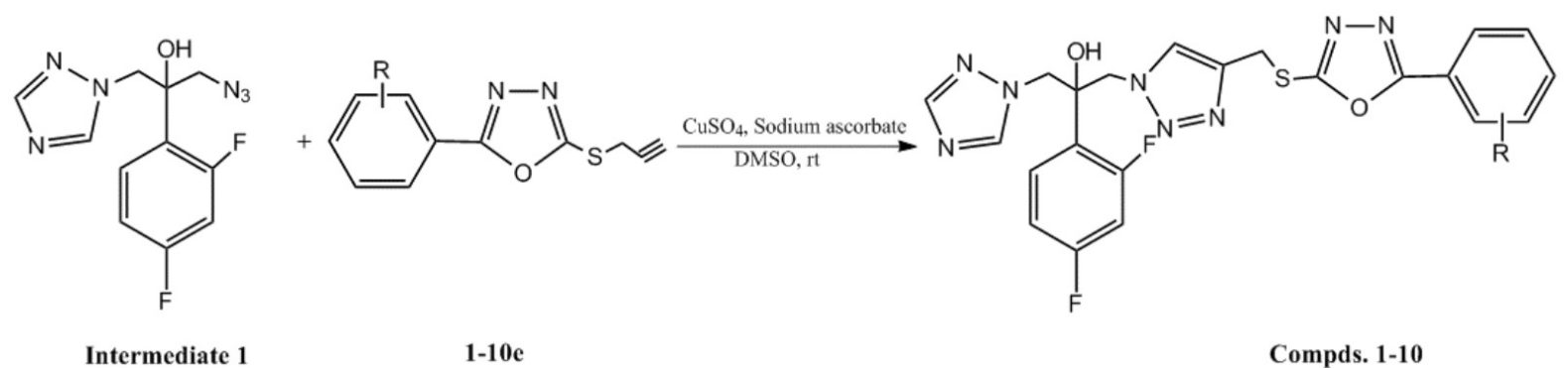

Scheme-I: Synthesis of the target compounds 1-10

$6.69(\mathrm{~m}, 2 \mathrm{H}), 5.36(\mathrm{~s}, 1 \mathrm{H}), 4.88-4.84(\mathrm{~d}, 1 \mathrm{H}, J=14.3 \mathrm{~Hz})$, 4.78-4.62 (m, 2H), 4.61-4.42 (m, 2H), 4.33-4.29 (d, 1H, J = 14.1 Hz), $2.43(\mathrm{~s}, 3 \mathrm{H}) ;[\mathrm{M}+\mathrm{H}]^{+}$511.14, found 511.64.

Compound 3: Yellow oil, yield: $86 \%$; ${ }^{1} \mathrm{H}$ NMR $(300$ $\left.\mathrm{MHz}, \mathrm{CDCl}_{3}\right) \delta 8-7.80(\mathrm{~m}, 5 \mathrm{H}), 7.68-7.60(\mathrm{~m}, 1 \mathrm{H}), 7.52-7.27$ $(\mathrm{m}, 1 \mathrm{H}), 6.87-6.66(\mathrm{~m}, 4 \mathrm{H}), 4.87-4.86(\mathrm{~m}, 1 \mathrm{H}), 4.79-4.69(\mathrm{~m}$, $3 \mathrm{H}), 4.60-4.47$ (m, 1H), 3.71-3.67 (d, 1H, $J=12.8 \mathrm{~Hz}), 3.56-$ $3.51(\mathrm{~d}, 1 \mathrm{H}, J=12.8 \mathrm{~Hz}), 2.38(3 \mathrm{H}, \mathrm{s}) ;[\mathrm{M}+\mathrm{H}]^{+} 511.14$, found 511.51 .

Compound 4: Pale yellow oil, yield: $88 \% ;{ }^{1} \mathrm{H}$ NMR (300 $\left.\mathrm{MHz}, \mathrm{CDCl}_{3}\right) \delta 8(\mathrm{~s}, 1 \mathrm{H}), 7.84(\mathrm{~s}, 1 \mathrm{H}), 7.75(\mathrm{~s}, 1 \mathrm{H}), 7.45-7.31$ $(\mathrm{m}, 3 \mathrm{H}), 6.85-6.62(\mathrm{~m}, 3 \mathrm{H}), 4.88-4.83(\mathrm{~d}, 1 \mathrm{H}, J=14.2 \mathrm{~Hz})$, 4.74-4.72 (d, $2 \mathrm{H}, J=6.5 \mathrm{~Hz}), 4.56-4.55(\mathrm{~d}, 2 \mathrm{H}, J=2.9 \mathrm{~Hz})$, 4.32-4.27 (d, $1 \mathrm{H}, J=14.3 \mathrm{~Hz}) ;[\mathrm{M}+\mathrm{H}]^{+} 586.09$, found 586.42 .

Compound 5: Yellow oil, yield: $82 \%$; ${ }^{1} \mathrm{H}$ NMR (300 $\left.\mathrm{MHz}, \mathrm{CDCl}_{3}\right) \delta 8.02$ (s, 1H), $7.86(\mathrm{~s}, 1 \mathrm{H}), 7.76$ (s, 1H), 7.47$7.31(\mathrm{~m}, 1 \mathrm{H}), 6.85-6.68(\mathrm{~m}, 2 \mathrm{H}), 4.89-4.84(\mathrm{~d}, 1 \mathrm{H}, J=14.1$ $\mathrm{Hz}), 4.79-4.67$ (d, 2H, $J=14.2 \mathrm{~Hz}), 4.61-4.56$ (d, 2H, $J=7.4$ $\mathrm{Hz}), 4.32-4.27(\mathrm{~d}, 1 \mathrm{H}, J=14.2 \mathrm{~Hz}) ;[\mathrm{M}+\mathrm{H}]^{+} 568.08$, found 568.35 .

Compound 6: Brown oil, yield: $81 \%$; ${ }^{1} \mathrm{H}$ NMR (300 $\left.\mathrm{MHz}, \mathrm{CDCl}_{3}\right) \delta$ 8.06-7.71 (4H, m), 7.52-7.29 (3H, m), 6.80$6.74(3 \mathrm{H}, \mathrm{m}), 5.18-5.16(1 \mathrm{H}, \mathrm{d}, J=6.2 \mathrm{~Hz}), 4.95-4.68(4 \mathrm{H}$, m), 4.56-4.54 (1H, d, $J=6 \mathrm{~Hz}), 2.63-2.61(2 \mathrm{H}, \mathrm{d}, J=6.1 \mathrm{~Hz})$, $1.68(2 \mathrm{H}, \mathrm{m}), 1.45-1.12(3 \mathrm{H}, \mathrm{m}) ;[\mathrm{M}+\mathrm{H}]^{+} 538.17$, found 538.55 .

Compound 7: Yellow oil, yield: $85 \%$; ${ }^{1} \mathrm{H}$ NMR (300 $\left.\mathrm{MHz}, \mathrm{CDCl}_{3}\right) \delta$ 8.11-7.74 (m, 4H), 7.52-7.29 (m, 3H), 6.82$6.74(\mathrm{~m}, 3 \mathrm{H}), 5.17-5.15(\mathrm{~d}, 1 \mathrm{H}, J=6.2 \mathrm{~Hz}), 4.85-4.69(\mathrm{~m}$, $4 \mathrm{H}), 4.57-4.53$ (d, $1 \mathrm{H}, J=12 \mathrm{~Hz}), 2.62-2.58$ (m, 2H), 1.68$1.64(\mathrm{~m}, 4 \mathrm{H}), 1.45-1.22(\mathrm{~m}, 3 \mathrm{H}) ;[\mathrm{M}+\mathrm{H}]^{+} 552.18$, found 552.57 .
Compound 8: Yellow oil, yield: $83 \%$; ${ }^{1} \mathrm{H}$ NMR (300 $\left.\mathrm{MHz}, \mathrm{CDCl}_{3}\right) \delta 8.01$ (s, 1H), $7.82(\mathrm{~s}, 1 \mathrm{H}), 7.74(1 \mathrm{H}, \mathrm{s}), 7.39-$ $7.31(\mathrm{dd}, 3 \mathrm{H}, J=15.5,9.1 \mathrm{~Hz}), 6.85-6.68(\mathrm{~m}, 3 \mathrm{H}), 4.86-4.81(\mathrm{~d}$, $1 \mathrm{H}, J=14.3 \mathrm{~Hz}), 4.74-4.73(\mathrm{~d}, 2 \mathrm{H}, J=2.8 \mathrm{~Hz}), 4.54-4.53$ (d, $2 \mathrm{H}, J=2.1 \mathrm{~Hz}), 4.34-4.29(\mathrm{~d}, 1 \mathrm{H}, J=14.3 \mathrm{~Hz}), 1.25(\mathrm{~s}, 9 \mathrm{H})$; $[\mathrm{M}+\mathrm{H}]^{+}$552.18, found 552.57.

Compound 9: Yellow oil, yield: $89 \%$; ${ }^{1} \mathrm{H}$ NMR (300 $\left.\mathrm{MHz}, \mathrm{CDCl}_{3}\right) \delta 8.14-8.11(\mathrm{~d}, 2 \mathrm{H}, J=8.1 \mathrm{~Hz}), 8.04(\mathrm{~s}, 1 \mathrm{H})$, $7.85(\mathrm{~s}, 1 \mathrm{H}), 7.84-7.73(\mathrm{~m}, 3 \mathrm{H}), 7.37(\mathrm{~m}, 1 \mathrm{H}), 6.82-6.65(\mathrm{~m}$, $2 \mathrm{H}), 4.89-4.84(\mathrm{~d}, 1 \mathrm{H}, J=14.4 \mathrm{~Hz}), 4.80-4.69$ (q, $2 \mathrm{H}, J=$ $14.3 \mathrm{~Hz}), 4.56$ (s, 2H), 4.35-4.31 (d, 1H, J = 14.2 Hz), 2.63$2.60(\mathrm{~m}, 2 \mathrm{H}), 1.66-1.65(\mathrm{~m}, 6 \mathrm{H}), 1.45-1.15(\mathrm{~m}, 3 \mathrm{H}) ;[\mathrm{M}+\mathrm{H}]^{+}$ 566.20, found 566.62.

Compound 10: Yellow oil, yield: $80 \%$; ${ }^{1} \mathrm{H}$ NMR (300 $\left.\mathrm{MHz}, \mathrm{CDCl}_{3}\right) \delta$ 8.0-7.96 (d, 3H, $\left.J=13.4 \mathrm{~Hz}\right), 7.83-7.76(\mathrm{~m}$, 2H), 7.61-7.42 (m, 3H), 7.39-7.31(m, 1H), 6.86-6.55 (m, 2H), $5.46(\mathrm{~s}, 1 \mathrm{H}), 4.87-4.82(\mathrm{~d}, 1 \mathrm{H}, J=14.3 \mathrm{~Hz}), 4.77-4.63(\mathrm{~m}$, $2 \mathrm{H}), 4.53(\mathrm{~s}, 1 \mathrm{H}), 4.35-4.40(\mathrm{~d}, 1 \mathrm{H}, J=14.3 \mathrm{~Hz}) ;[\mathrm{M}+\mathrm{H}]^{+}$ 496.12, found 496.43 .

\section{RESULTS AND DISCUSSION}

All the title compounds (1-10) were screened for antifungal activity against eight pathogenic fungi. The in vitro minimal inhibitory concentrations (MICs) of the compounds were determined by the micro-broth dilution method in 96well microtest plates according to the methods defined by the National Committee for Clinical Laboratory Standards $\left(\right.$ NCCLS) ${ }^{15}$. Itraconazole (ICZ) and fluconazole (FCZ) served as the positive controls were obtained from their respective manufacturers. Table-1 showed that all the synthesized compounds show moderate activity against all the tested fungal pathogens. Most of our compounds showed the good antifungal 


\begin{tabular}{|c|c|c|c|c|c|c|c|c|c|}
\hline \multicolumn{10}{|c|}{$\begin{array}{c}\text { TABLE-1 } \\
\text { ANTIFUNGAL ACTIVITY OF THE TARGET COMPOUNDS in vitro }\left(\mathrm{MIC}_{80}: \mu \mathrm{g} / \mathrm{mL}\right)\end{array}$} \\
\hline Compd. & $\mathrm{R}$ & C. alb Y0109 & C. alb SC5314 & C. pra & C. neo & C. gla & A. fum & T. rub & M. gyp \\
\hline 1 & $2-\mathrm{CH}_{3}$ & 0.5 & 0.5 & 0.5 & 0.5 & 0.5 & $>64$ & 64 & $>64$ \\
\hline 2 & $3-\mathrm{CH}_{3}$ & 1 & 2 & 1 & 0.5 & 1 & $>64$ & 32 & 32 \\
\hline 3 & $4-\mathrm{CH}_{3}$ & 1 & 4 & 2 & 1 & 0.25 & 64 & 8 & 16 \\
\hline 4 & $3,5-\mathrm{NO}_{2}$ & 1 & 1 & 1 & 2 & 2 & $>64$ & 64 & 64 \\
\hline 6 & 4- $\left(\mathrm{CH}_{2}\right)_{2} \mathrm{CH}_{3}$ & 0.5 & 0.5 & 8 & 8 & 0.5 & $>64$ & 32 & 64 \\
\hline 7 & 4- $\left(\mathrm{CH}_{2}\right)_{3} \mathrm{CH}_{3}$ & 2 & 2 & 16 & 8 & 2 & $>64$ & $>64$ & $>64$ \\
\hline 8 & $4-\mathrm{C}\left(\mathrm{CH}_{2}\right)_{3}$ & 4 & 2 & 4 & 2 & 0.5 & $>64$ & 32 & 32 \\
\hline 9 & 4- $\left(\mathrm{CH}_{2}\right)_{4} \mathrm{CH}_{3}$ & 8 & 8 & 16 & 4 & 1 & 32 & 16 & 16 \\
\hline 10 & $\mathrm{H}$ & 1 & 4 & 4 & 0.5 & 1 & $>64$ & $>64$ & $>64$ \\
\hline ICZ & - & 8 & 4 & 8 & 4 & 8 & 8 & 2 & 2 \\
\hline $\mathrm{FCZ}$ & - & 0.5 & 0.5 & 1 & 1 & 1 & $>64$ & 8 & 8 \\
\hline
\end{tabular}

Abbreviations: C. alb. Y0109 = Candida albicans Y0109; C. alb. SC5314 = Candida albicans SC5314; C. par. $=$ Candida parapsilosis; C. neo. $=$ Cryptococcus neoformans; C. gla. = Candida glabrata; A. fum. = Aspergillus fumigatus; . rub. = Trichophyton rubrum; M. gyp. = Microsporum gypseum. ICZ = Itraconazole; FCZ = Fluconazole.

activities than the positive control itraconazole. It is noted that the $\mathrm{MIC}_{80}$ values of compounds $\mathbf{1}$ and $\mathbf{6}$ were equal or a little bit lower than that of fluconazole and itraconazole against $C$. albicans, C. parapsilosis, C. neoformans and C. glabrata.

\section{Conclusion}

In summary, a novel series of antifungal agents have been successfully designed and synthesized. Antifungal activity assay in vitro indicates that most of the compounds showed moderate antifungal activities against systemic pathogenic fungi. Some compounds (such as compd. 1 and 6) show highly antifungal activity with broad spectrum, which were valuable for further studies.

\section{ACKNOWLEDGEMENTS}

This work was supported by the National Natural Science Foundation of China (Nos. 20772153), the Creativity and Innovation Training Program of Second Military Medical University (Grant No. ZD2013029).

\section{REFERENCES}

1. P.G. Pappas, B.D. Alexander, D.R. Andes, S. Hadley, C.A. Kauffman, A. Freifeld, E.J. Anaissie, L.M. Brumble, L. Herwaldt, J. Ito, D.P. Kontoyiannis, G.M. Lyon, K.A. Marr, V.A. Morrison, B.J. Park, T.F. Patterson, T.M. Perl, R.A. Oster, M.G. Schuster, R. Walker, T.J. Walsh, K.A. Wannemuehler and T.M. Chiller, Clin. Infect. Dis., 50, 1101 (2010).

2. M.D. Richardson, J. Antimicrob. Chemother., 56(suppl 1), 5 (2005).

3. D.W. Warnock, Nippon Ishinkin Gakkai Zasshi, 48, 1 (2007).

4. C. Garcia-Vidal, D. Viasus and J. Carratalà, Curr. Opin. Infect. Dis., 26, 270 (2013).

5. M.H. Miceli, J.A. Díaz and S.A. Lee, Lancet Infect. Dis., 11, 142 (2011).

6. J.R. Wingard and H. Leather, Biol. Blood Marrow Transplant, 10, 73 (2004).

7. S.K. Fridkin and W.R. Jarvis, Clin. Microbiol. Rev., 9, 499 (1996).

8. M.A. Pfaller, Am. J. Med., 125, S3 (2012).

9. D.S. Johnson and J.J. Li, The Art of Drug Synthesis, 7, 71 (2007).

10. M. Canuto and F. Rodero, Lancet Infect. Dis., 2, 550 (2002).

11. T. Konosu, Y. Tajima, N. Takeda, T. Miyaoka, M. Kasahara, H. Yasuda and S. Oida, Chem. Pharm. Bull. (Tokyo), 38, 2476 (1990).

12. S.C. Yu, X.Y. Chai, H. Hu, Y. Yan, Z. Guan, Y. Zou, Q. Sun and Q. Wu, Eur. J. Med. Chem., 45, 4435 (2010).

13. B.G. Wang, S.C. Yu, X.Y. Chai, Y.Z. Yan, H.G. Hu and Q.Y. Wu, Chin. Chem. Lett., 22, 519 (2011).

14. Y. Zou, Q. Zhao, J. Liao, H. Hu, S. Yu, X. Chai, M. Xu and Q. Wu, Bioorg. Med. Chem. Lett., 22, 2959 (2012).

15. National Committee for Clinical Laboratory Standards, Reference Method for Broth Dilution Antifungal Susceptibility Testing of Yeasts Approved standard. Document M27-A2, National Committee for Clinical Laboratory Standards, Wayne, PA (2002). 